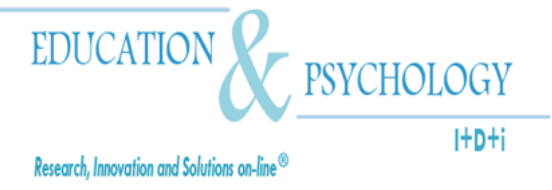

\title{
Percepciones y actitudes del alumnado extranjero y del profesorado: un estudio empírico en las aulas de acogida de Cataluña
}

\author{
Carina Siqués ${ }^{1}$, Ignasi Vila ${ }^{2}$, Santiago Perera ${ }^{2}$ \\ ${ }^{1}$ Departamento de Didácticas Específicas, Universidad de Girona \\ ${ }^{2}$ Departamento de Psicología, Universidad de Girona
}

\section{España}




\section{Resumen}

Introducción. La investigación que presentamos intenta conocer las percepciones y las actitudes que el alumnado de origen extranjero que acude a las aulas de acogida de primaria de Cataluña y su profesorado tienen sobre la institución escolar, sobre su implicación en las prácticas escolares y sobre las relaciones que se allí se establecen.

Método. Las percepciones fueron obtenidas mediante tres cuestionarios. Cada cuestionario consta de cuatro apartados: adaptación al medio escolar, estrategias de aprendizaje, convivencia y relación con los pares y con los adultos. Un cuestionario se administró a 5724 estudiantes extranjeros que se habían incorporado al sistema educativo de Cataluña desde el año 2004 y que utilizaban el aula de acogida durante el curso 2006-2007. Los otros dos cuestionarios fueron administrados respectivamente a su profesorado tutor del aula ordinaria y del aula de acogida. La administración se realizó durante los meses de mayo y junio de 2007 y todos los datos fueron incorporados al programa SPSS versión 15. Se utilizaron dos variables del alumnado extranjero: familia lingüística y continente de procedencia. Se realizó un análisis factorial de las respuestas de los tres cuestionarios y, posteriormente, hicimos un análisis ANOVA de cada factor obtenido con las variables seleccionadas del alumnado.

Resultados. Los resultados muestran que no existe coincidencia entre las percepciones subjetivas del alumnado africano y asiático sobre su implicación en la institución escolar y las percepciones que tiene el profesorado de dicho alumnado. Al contrario, existe una importante coincidencia entre ambas percepciones cuando el alumnado extranjero procede de los países del Este o de la Europa Comunitaria. Respecto al alumnado latinoamericano, prevalecen los acuerdos más que los desacuerdos en ambas percepciones. Finalmente, los datos muestran que el profesorado percibe significativamente al alumnado africano y asiático con más problemas de integración que al alumnado europeo o latinoamericano.

Discusión y conclusiones. El artículo discute las percepciones mutuas del alumnado y del profesorado a partir de las experiencias educativas previas del alumnado y las percepciones sociales sobre la integración de los diferentes colectivos de personas extranjeras y, a la vez, concluye que las percepciones que tiene el profesorado sobre el alumnado extranjero coinciden en general con los estereotipos y los prejuicios existentes en la sociedad sobre las personas extranjeras.

Palabras Clave: actitudes escolares, educación intercultural, aulas de acogida, diversidad lingüística y étnica, alumnado extranjero.

Recibido: 09/07/08 Aceptación inicial: 27/07/08 Aceptación final: 11/11/08 


\begin{abstract}
Introduction. The research being presented tries to find out the perceptions and attitudes foreign school students attending linguistic support classes in primary school and their teachers have about the school institution, the students' implication in school practices and the relationships established.
\end{abstract}

Method. The perceptions were obtained through the administration of three questionnaires each of which had four differentiated sections: adaptation to the school setting, learning strategies, relationship with school peers and relationship with adults. One of the questionnaires was completed by 5724 foreign students who had incorporated into the school system in Catalonia from year 2004 and who were using the linguistic support classroom during academic year 2006-2007. The other two questionnaires were completed by the mainstream classroom teachers and by the linguistic support teachers respectively during the months of May and June 2007. SPSS15 was used to analyse the data. Two different variables were used with foreign students: linguistic family and continent of origin. With the answers to the three questionnaires we did a factorial analysis and an ANOVA analysis of each factor obtained with the variables of the students selected.

Results. The results show that there is no coincidence between the subjective perceptions Asian and African students have and those of their teachers. On the contrary, the perceptions of students and teachers coincide with students from Eastern and Community Europe. With Latin American students, there are more coincidences than discrepancies between students and teachers. Finally, the data shows that teachers significantly perceive African and Asian students having more problems of integration than European and Latin American students.

Discussion and conclusions. The article discusses the mutual perceptions of students and teachers through the students' previous school experiences and the social perceptions about the integration of different groups of foreign people and, at the same time, concludes that teachers' perceptions towards foreign students generally coincide with stereotypes and prejudices existing in society about foreign people.

Keywords: school attitudes; intercultural education; linguistic support classes; linguistic and ethnic diversity, migrant students.

Received: 07/09/08 Initial Acceptance: 07/27/08_ Final Acceptance: 11/11/08 


\section{Introducción}

La educación intercultural se ha instalado con fuerza en el horizonte educativo (Banks y Banks, 1989; Besalú y Vila, 2007; Cummins, 2000; Fernández-Castillo, 2005). Su propósito es la promoción desde las aulas de actitudes y valores que garanticen la cohesión social en sociedades cada vez más multiculturales y plurilingües. Por eso, la educación intercultural está dirigida a todo el alumnado, independientemente de su origen nacional. Sin embargo, la práctica de la educación intercultural comporta necesariamente que el profesorado tenga unas teorías implícitas acerca de la bondad de la escuela inclusiva o de la heterogeneidad en las aulas escolares y, a la vez, una concepción sobre la infancia y la adolescencia que no esté encorsetada por prejuicios y estereotipos étnicos, lingüísticos o religiosos.

Desde 1990, diferentes trabajos (Mcintosh, 1990; Sleeter, 1992) han evidenciado que una parte del profesorado tiene prejuicios y estereotipos respecto a las minorías étnicas y lingüísticas. Por eso, y de acuerdo con algunos principios de la educación intercultural, se han desarrollado programas de formación del profesorado dirigidos al desarrollo de actitudes positivas hacia la multiculturalidad y el plurilingüismo (Mcintyre, 1997; Weiner, 2000). En la década del 2000, diferentes estudios han mostrado que en Europa crecen los prejuicios y las actitudes negativas hacia las minorías étnicas. Verbeck, Scheepers y Felling (2002) emplean la distinción entre actitudes "tradicionales" abiertas y actitudes "contemporáneas" encubiertas (Coenders, Scheepers, Sniderman y Verbeck, 2001) hacia las minorías étnicas para comprender la naturaleza, el lugar social, los determinantes y las consecuencias de las actitudes abiertamente negativas de la sociedad holandesa hacia las minorías étnicas.

La existencia de actitudes sociales negativas hacia las minorías étnicas y, más en concreto, hacia las personas de origen extranjero ha suscitado investigaciones sobre la existencia o no de prejuicios y estereotipos hacia el alumnado inmigrante en la educación escolar. Algunos trabajos (Solomona, Portelli, Beverly-Jean y Campbell, 2005) evidencian que existen importantes prejuicios por parte del profesorado e, incluso, se ha mostrado que, en algunas ocasiones, hay una estrecha relación entre los prejuicios del profesorado y su práctica educativa. Primo, Quaglia y Schavo (2008) analizan en Italia los prejuicios de 79 maestros hacia 396 niños y niñas de origen extranjero. Los datos muestran la existencia de importantes prejuicios que además operan negativamente en la relación profesor-alumno, especialmente en situacio- 
nes de conflicto, lo cual conlleva percepciones y evaluaciones desajustadas por parte del profesorado.

Los estudios sociológicos realizados en España sobre las actitudes de la población hacia el fenómeno migratorio y las personas de origen extranjero muestran que, en los últimos años, crecen, al igual que en otros países europeos, los estereotipos y los prejuicios (European Monitoring Centre on Racism and Xenophobia, 2005). Ciertamente, España es probablemente el país europeo en el que el porcentaje de población que manifiesta actitudes negativas hacia la inmigración es más bajo y en el que la población manifiesta más tolerancia y solidaridad con las personas de origen extranjero (INE, 2008). Pero, no cabe duda que la marea de la islamofobia y el racismo comienza a tener un hueco en las representaciones sociales de las personas españolas, especialmente en aquellas zonas en las que el porcentaje de población de origen extranjero se ha incrementado espectacularmente en los últimos diez años.

El profesorado no es ajeno a la modificación de las percepciones sociales y, por eso, podemos pensar que, igual que en el resto de las sociedades occidentales, una parte asume también los prejuicios y los estereotipos sobre la inmigración y sobre las personas de origen extranjero y, en consecuencia, también sobre la infancia y la adolescencia de origen extranjero. Sin embargo, los estudios empíricos en España sobre las actitudes del profesorado que permitan poder desarrollar una práctica acorde con la educación intercultural son prácticamente inexistentes. De ellos cabe destacar la investigación de León del Barco, Mira y GómezCarroza (2007) sobre las actitudes del alumnado de magisterio de las universidades de Extremadura (España) y Évora (Portugal) hacia la creciente multiculturalidad de las aulas escolares y hacia la inmigración. Sus conclusiones afirman que globalmente no existen prejuicios ni estereotipos hacia la educación intercultural o hacia el alumnado de origen extranjero. No obstante, la autoría del artículo afirma que el alumnado de magisterio que evita el contacto con las personas de procedencia extranjera y que mantiene actitudes negativas hacia su personalidad también tiene actitudes negativas respecto a su presencia en las aulas y hacia la educación intercultural.

Pero, independientemente de las percepciones y las representaciones subjetivas del profesorado, la realidad multicultural y plurilingüe de las aulas se impone día a día. En Cataluña, cerca del 14\% del alumnado de la educación obligatoria es extranjero y la cifra aumenta año tras año. Además, en dicha cifra no se contabiliza al alumnado de procedencia extranjera 
que ya ha nacido en Cataluña y que representa también un número muy importante. Así, en el año 2006, más del 25\% de los recién nacidos en Cataluña tenían el padre o la madre, o ambos, de origen extranjero (IDESCAT, 2008).

Los partidos de izquierda que ganaron las elecciones autonómicas en Cataluña en el año 2004, el llamado gobierno tripartito, iniciaron una política educativa basada en el plan LIC “Llengua, Interculturalitat i Cohesió Social” (Departament d'Educació, 2004). El plan LIC (Besalú y Vila, 2005, 2007; Besalú, 2006; Serra, 2006) fue diseñado para garantizar la cohesión social y está dirigido a todo el alumnado de Cataluña en general pero contempla medidas específicas para el alumnado de origen extranjero. Su andadura se inició el curso 2004-2005 y uno de sus pilares fue la creación de las aulas de acogida en todos los centros escolares subvencionados con fondos públicos que escolarizaban a alumnado extranjero de nueva incorporación al sistema educativo. En el curso 2006-2007 existían ya en Cataluña 1050 aulas de acogida repartidas en centros de primaria y de secundaria.

El aula de acogida es un recurso que ofrece la administración a los centros escolares y que consiste en dotar al centro con una persona más en plantilla y dar una aportación material tanto en recursos como económica. Hay centros que escolarizan a un gran número de alumnado extranjero en los que existe más de un aula de acogida y éstos reciben los mismos recursos para cada una de las aulas que poseen. El uso que cada centro escolar hace del aula de acogida es decidido por el claustro de profesores y profesoras. Algunos centros optan por ofrecer apoyo lingüístico al alumnado de origen extranjero en el aula ordinaria mientras que la gran mayoría optan por ofrecer dicho apoyo en un espacio físico distinto que posee el centro escolar y al que acude el alumnado durante una o dos horas al día. Los usos escolares del aula de acogida varían enormemente, entre otras variables, en función del tiempo de estancia en Cataluña del alumnado, de su lengua propia o de su escolarización previa (Siqués, 2008; Vila, Canal, Mayans, Perera, Serra y Siqués, 2007; Vila, Canal, Mayans, Perera, Serra y Siqués, en prensa; Vila, Perera y Serra, 2006).

Durante el curso 2006-2007 se realizó una evaluación de las aulas de acogida de los centros de educación primaria ${ }^{1}$ de Cataluña (Siqués, 2008). La evaluación utilizó dos instrumentos: una prueba de competencias básicas en lengua catalana (usuario A2 de acuerdo con la

\footnotetext{
${ }^{1}$ La Educación Primaria en España es obligatoria y consta de seis cursos. En el primero se escolariza el alumnado que a lo largo del año cumple siete años.
} 
nomenclatura del Consejo de Europa) y una prueba de adaptación escolar. Se introdujo la prueba de adaptación escolar porque, en la medida en que la lengua se aprende a través de hacer cosas con ella, mayores grados de adaptación escolar comportarían mayores implicaciones en hacer cosas con la lengua escolar y, por tanto, redundaría en un mayor conocimiento del catalán.

La prueba de adaptación escolar fue respondida por el alumnado de origen extranjero que utilizaba el aula de acogida, por el profesorado tutor del aula ordinaria donde estaba escolarizado el alumnado y por el profesorado del aula de acogida. Dicha prueba, entre otras cosas, servía para evaluar las representaciones que el alumnado de origen extranjero y el profesorado tenían sobre la institución escolar, así como para evaluar las prácticas que allí se realizaban y las relaciones que se establecían. Es decir, la prueba pretendía poder ser un buen instrumento para conocer los acuerdos y las discrepancias entre el profesorado y el alumnado de origen extranjero acerca de la institución escolar y de la implicación del alumnado.

En definitiva, el análisis de las respuestas del alumnado de origen extranjero y del profesorado al cuestionario de adaptación escolar permite hacer una aproximación a las teorías implícitas del profesorado sobre el alumnado de origen extranjero, así como valorar sus actitudes sobre su escolarización.

\section{Método}

\section{Participantes}

Los participantes fueron 5724 alumnas y alumnos de origen extranjero que se habían ido incorporando al sistema educativo de Cataluña desde el año 2004 y que acudían al aula de acogida durante el curso 2006-2007 así como el profesorado tutor del aula ordinaria y del aula de acogida de los 525 centros de educación primaria que escolarizaban a este alumnado.

Las edades del alumnado variaban entre los 5 y los 13 años. Así, un $1.8 \%$ tenía de 5 a 7 años, un $18.3 \%$ tenía 8 años, un $22.3 \%$ tenía 9 años, un $25.3 \%$ tenía 10 años, un $25{ }^{\circ} 4 \%$ tenía 11 años y un 6’7\% tenía de 12 a 13 años. En el ciclo inicial (5-7 años) sólo se escolarizaba un 
0`9\% de alumnado y el resto se repartía casi a partes iguales entre el ciclo medio (46.2\%) y el ciclo superior $(51.8 \%)$.

\section{Instrumentos}

La prueba de adaptación escolar la forman tres cuestionarios que responden el alumnado, el profesorado tutor del aula ordinaria y el profesorado del aula de acogida respectivamente. Con el conjunto de los tres cuestionarios obtenemos una puntuación única. La base sobre la cual se fundamenta la prueba de adaptación escolar utilizada contempla cuatro aspectos diferenciados:

1. Adaptación al medio escolar: se trata de saber si el alumnado ha alcanzado un conocimiento básico del centro (espacios, distribución temporal, costumbres, hábitos, tipo de tareas, etc.) y ha desarrollado un grado de confianza suficiente en el nuevo entorno que le permite sentirse cómodo y seguro y, por tanto, poder afrontar los procesos de enseñanza y aprendizaje y de socialización sin inquietudes ni miedos que podrían interferir en los aprendizajes.

2. Estrategias para el aprendizaje: el objetivo es determinar si el alumnado tiene las estrategias para afrontar, con posibilidades de éxito, las tareas escolares que se le piden. Es importante diferenciar estas estrategias básicas (hábitos de trabajo, interés, participación, atención, esfuerzo, capacidad de solicitar ayuda, etc.) de los conocimientos.

3. Convivencia y relación entre iguales: se trata de detectar los puntos fuertes y los puntos débiles en la red de relaciones que establece el alumnado con sus iguales y detectar posibles actitudes de rechazo y ensimismamiento.

4. Convivencia y relación con los adultos: el objetivo es conocer el tipo de relación que el alumnado ha establecido con las personas adultas, especialmente con el profesorado.

La preguntas al cuestionario contestado por el alumnado hacen referencia a su satisfacción en el aula de acogida y en el aula ordinaria, a su interés por las actividades que se realizan en las dos aulas y a su participación, a su percepción sobre el profesorado y a las relaciones que establece con el resto del alumnado en las dos aulas. 
Los cuestionarios contestados por la persona encargada del aula de acogida y por la persona tutora del aula ordinaria constan de cuatro apartados: actitud general del alumno, actitud ante el trabajo escolar, interacciones con el resto del alumnado e interacciones con el profesorado y otras personas profesionales de la educación del centro escolar.

En cada pregunta (por ejemplo, "trabaja en el aula de acogida") hay cuatro respuestas que van de mucho (4) a nada (1). Cada alumno obtiene una puntuación que es el resultado de sumar las puntuaciones que dan a cada ítem el alumnado y el profesorado del aula de acogida y del aula ordinaria en los tres cuestionarios. Hay cuatro preguntas que restan como, por ejemplo, "falta a clase" o "únicamente se relaciona con el alumnado de su propia etnia". La puntuación mínima de la prueba es 50 y la máxima 208.

La fiabilidad de los tres cuestionarios fue medida mediante el Alfa de Cronbach con los valores siguientes: 0,783 para el cuestionario del alumnado; 0,845 para el cuestionario del aula de acogida y 0,859 para el cuestionario del profesorado del aula ordinaria.

\section{Variables}

Las lenguas habladas por los 5724 participantes son 58 y sus países de origen son 94. Hemos utilizado ambas variables para establecer grupos diferentes de alumnas y alumnos y, así, poder analizar de forma diferencial las actitudes del alumnado y del profesorado en relación a la institución escolar y la existencia o no de perjuicios por parte del profesorado hacia los diferentes grupos.

No obstante, dado el elevado número de lenguas habladas así como de países de origen del alumnado, hemos transformado ambas variables en dos nuevas: familia lingüística y continente.

1. Familia lingüística.

1. Lenguas indoeuropeas románicas (castellano, rumano, francés, italiano y portugués).

2. Lenguas indoeuropeas eslavas (ruso, polaco, búlgaro, ucraniano, checo, esloveno, eslovaco y serbocroata). 
3. Lenguas afroasiáticas semíticas (árabe).

4. Lenguas indoeuropeas germánicas (inglés, holandés, alemán, islandés, sueco y noruego).

5. Lenguas sinotibetanas siníticas (todas las variantes del chino).

6. Lenguas nigerocongolesas mandé (bambara, soninke, malinké y mandinga).

7. Lenguas nigerocongolesas atlánticas (wolof y fula).

8. Lenguas austronésicas malayopolinésicas (tagalo y malgache).

9. Lenguas indoeuropeas indoiranianas (panjabi, urdu, bengalí, nepalí y hindi).

10. Lenguas amazig.

2. Continente.

Las dimensiones son Asia (1), América Central y del Sur (2), Europa del Este (3), África (4) y Europa (5).

\section{Procedimiento}

La prueba fue administrada al alumnado de las aulas de acogida que llevaba como mínimo seis meses en Cataluña y que no presentaba ninguna característica conductual de riesgo biológico. El profesorado tutor del aula de acogida y del aula ordinaria contestó los cuestionarios correspondientes. La dirección de los centros escolares suministró los datos del alumnado referidos a su lengua inicial y su país de origen.

Las pruebas fueron administradas entre finales de mayo y mediados de junio del año 2007 por el profesorado del aula de acogida o por las personas asesoras del programa LIC del Departament d'Educació de la Generalitat de Cataluña a lo largo de una semana y fueron enviadas vía telemática al Servei d'Ensenyament del Català (SEDEC) del Departament d’Educació. El envío se hizo de manera ordenada para evitar bajadas de tensión en la red y no perder ningún participante. 
El cuestionario de adaptación escolar así como las instrucciones para su correcta administración están a disposición de todo el profesorado en la web del programa LIC (www.xtec.cat/lic/index.htm).

Todos los datos fueron introducidos en el programa SPSS versión 15.

\section{Análisis de los datos}

Se realizó un análisis factorial con método de extracción de componentes principales y rotación Varimax con Kaiser de cada una de las tres partes del cuestionario. La validez del análisis comportaba que el determinante fuera mayor que cero. Además, utilizamos las pruebas KMO y esferidad de Barlett para verificar la idoneidad del análisis factorial. Posteriormente, se utilizó el análisis de varianza con los factores obtenidos y las variables familia lingüística y continente.

Dado que en la variable "lengua románica" se incluye el castellano que es la lengua que habla cerca del $40 \%$ de la muestra, hemos separado este alumnado, designado como hispano o latinoamericano, del resto del alumnado de lengua románica y hemos comparado sus resultados especialmente con el alumnado de lengua rumana que, por otra parte, agrupa a la mayor parte del resto del alumnado de lengua románica (65\%).

\section{Resultados}

Análisis factorial de componentes principales de las preguntas del cuestionario.

A continuación se presentan los tres análisis factoriales correspondientes a las respuestas al cuestionario del alumnado (1), del profesorado del aula de acogida (2) y del profesorado del aula ordinaria (3). Se ha utilizado la matriz de correlaciones para determinar los componentes y la matriz de componentes rotados de acuerdo con la normalización de Varimax con Kaiser. Las pruebas KMO para los tres análisis factoriales fueron respectivamente 0.865 , 0.940 y 0.934 . Y, en relación con la esferidad de Barlett fueron respectivamente Chicuadrado=35863.711, gl=351, $<<0.001$; Chi-cuadrado=76668,160, gl=190, p <0’001 i Chicuadrado $=94763.237, \mathrm{gl}=253, \mathrm{p}<0.001$. 
El análisis factorial de las respuestas del alumnado al cuestionario.

El análisis factorial de las respuestas del alumnado al cuestionario especifica siete componentes que explican el $52.70 \%$ de la varianza. El primer componente lo hemos denominado "satisfacción en el aula ordinaria y esfuerzo individual" y contiene preguntas referidas a la satisfacción en el aula ordinaria ("me gusta lo que hacemos en el aula ordinaria" o "trabajo en el aula ordinaria") y al esfuerzo individual en el conjunto del trabajo escolar ("me esfuerzo por hacer las cosas mejor en el aula de acogida" o "me esfuerzo por hacer las cosas mejor en el aula ordinaria"). Explica el 20,639\% de la varianza. El segundo componente fue designado como "profesorado" y se refiere a la percepción del alumnado sobre el trabajo del profesorado ("el profesorado me escucha en el aula de acogida" o "el profesorado me ayuda en el aula ordinaria"). Explica el $6.561 \%$ de la varianza. El tercer componente lo denominamos "aprendizaje" e incluye las preguntas del cuestionario mediante las cuales el alumnado evalúa subjetivamente su proceso de aprendizaje ("puedo hacer los trabajos que me piden en el aula ordinaria" o "estoy aprendiendo en el aula ordinaria"). Explica el 6.474\% de la varianza. El cuarto componente ha sido designado como "satisfacción en el aula de acogida" e incluye las mismas preguntas sobre el aula de acogida que las que incluye el primer componente sobre el aula ordinaria. Explica el 5.361\% de la varianza.

Al quinto componente lo llamamos "preferencias en la relación con los compañeros" y se refiere al tipo de preferencias -alumnado de la propia cultura, alumnado del aula de acogida o todo tipo de alumnado- que el alumnado manifiesta en las relaciones con sus iguales. Explica el 5.098\% de la varianza. El sexto componente, denominado "compañeros y ayuda", especifica si el alumnado solicita y recibe ayuda del resto de iguales en el aula de acogida y el aula ordinaria. Explica el $4.683 \%$ de la varianza. Finalmente, el séptimo componente, "malestar con los compañeros", se refiere únicamente a dos preguntas: "hay compañeros que me molestan en el aula de acogida" y "hay compañeros que me molestan en el aula ordinaria"). Explica el 3.888\% de la varianza. Ver Tabla 1. 
Tabla 1. Matriz de componentes rotados del análisis factorial de las respuestas del alumnado al cuestionario de adaptación escolar. Educación primaria. Curso 2006-2007.

\begin{tabular}{|c|c|c|c|c|c|c|c|}
\hline & \multicolumn{7}{|c|}{ Componentes } \\
\hline & 1 & 2 & 3 & 4 & 5 & 6 & 7 \\
\hline $\begin{array}{l}\text { Estoy bien en el aula de aco- } \\
\text { gida } \\
\text { Me gusta lo que hacemos } \\
\text { Tengo ganas de trabajar } \\
\text { Entiendo lo que me explican } \\
\text { Estoy aprendiendo } \\
\text { Me esfuerzo en hacer las co- } \\
\text { sas mejor } \\
\text { Me gusta trabajar con... } \\
\text { Hay compañeros que me } \\
\text { ayudan } \\
\text { Hay compañeros que me mo- } \\
\text { lestan } \\
\text { Los profesores me escuchan } \\
\text { Los profesores me ayudan } \\
\text { Estoy bien en las clases del } \\
\text { aula ordinaria } \\
\text { Me gusta lo que hacemos } \\
\text { Estoy bien en el recreo } \\
\text { Trabajo } \\
\text { Tengo ganas de trabajar } \\
\text { Entiendo lo que me explican } \\
\text { Puedo hacer los trabajos que } \\
\text { me piden } \\
\text { Pido ayuda } \\
\text { Estoy aprendiendo } \\
\text { Me esfuerzo por hacer las } \\
\text { cosas mejor } \\
\text { En el recreo, me gusta estar } \\
\text { con... } \\
\text { En el aula, me gusta estar } \\
\text { con... } \\
\text { Hay compañeros que me } \\
\text { ayudan } \\
\text { Hay compañeros que me mo- } \\
\text { lestan } \\
\text { El profesorado me escucha } \\
\text { El profesorado me ayuda }\end{array}$ & $\begin{array}{l}\text {,505 } \\
\text { 714 } \\
\text {,719 }\end{array}$ & $\begin{array}{l}, 592 \\
, 553\end{array}$ & $\begin{array}{l}, 765 \\
500\end{array}$ & $\begin{array}{l}\text {,641 } \\
\text { 619 }\end{array}$ & $\begin{array}{l}\text { 814 } \\
\text {,813 }\end{array}$ & 533, & 801 \\
\hline
\end{tabular}

Análisis factorial de las respuestas del profesorado del aula de acogida al cuestionario.

El análisis factorial de las respuestas del profesorado del aula de acogida al cuestionario del alumnado especifica cuatro factores que explican el $63.544 \%$ de la varianza. 
El primer componente fue designado como "esfuerzo individual y hábitos de trabajo del alumnado" e incluye preguntas como "se esfuerza por hacer bien el trabajo escolar", "tiene interés por aprender" o "tiene hábitos de trabajo básicos". Explica el $42.279 \%$ de la varianza. El segundo componente lo denominamos "satisfacción del alumnado". Incluye preguntas como "se muestra se abierto" o "está bien adaptado al aula". Explica el 8.791\% de la varianza. El tercer componente es la antítesis del segundo componente ("se muestra agresivo" o "muestra actitudes de rechazo hacia determinados/as compañeros/as "). Lo hemos etiquetado como "malestar del alumnado". Explica el 6.954\% de la varianza. Finalmente, el cuarto componente expresa la relación del alumnado con sus iguales ("relación con los/las compañeros/as"). Explica el 5.520\% de la varianza. Ver Tabla 2.

Tabla 2. Matriz de componentes rotados del análisis factorial de las respuestas del profesorado del aula de acogida al cuestionario de adaptación escolar. Educación primaria. Curso 2006-2007.

\begin{tabular}{|c|c|c|c|c|}
\hline & \multicolumn{4}{|c|}{ Componentes } \\
\hline & 1 & 2 & 3 & 4 \\
\hline Se muestra seguro & & 820 & & \\
\hline Se muestra abierto & & ,717 & & \\
\hline Se muestra agresivo & & &,- 784 & \\
\hline Falta a clase & & & & \\
\hline Se muestra bien adaptado al aula & & 683, & & \\
\hline Tiene interés por aprender & 705, & & & \\
\hline Mantiene la atención & ,762 & & & \\
\hline Participa en las tareas que se proponen & ,611 & & & \\
\hline Se esfuerza por hacer bien el trabajo & ,746 & & & \\
\hline Sabe pedir ayuda & & ,495 & & \\
\hline Tiene los hábitos de trabajo básicos & ,837 & & & \\
\hline $\begin{array}{l}\text { Tiene las estrategias suficientes para afrontar } \\
\text { las actividades del aula }\end{array}$ & ,806 & & & \\
\hline Se relaciona con facilidad con los compañeros & & ,621 & & \\
\hline $\begin{array}{l}\text { Se relaciona, únicamente, con los compañeros } \\
\text { de la propia cultura }\end{array}$ & & & &,- 620 \\
\hline $\begin{array}{l}\text { Muestra actitudes de rechazo hacia determina- } \\
\text { dos compañeros }\end{array}$ & & &,- 720 & \\
\hline Es bien aceptado por los compañeros en el aula & & & & ,538 \\
\hline $\begin{array}{l}\text { Es bien aceptado por los compañeros en el re- } \\
\text { creo }\end{array}$ & & & & \\
\hline Tiene buena relación con el profesorado & & ,578 & & \\
\hline Actúa con autonomía & ,707 & & & \\
\hline Respeta las opiniones del profesorado & & & 621 & \\
\hline
\end{tabular}


Análisis factorial de las respuestas del profesorado del aula ordinaria al cuestionario.

El análisis factorial de las respuestas del profesorado del aula de acogida al cuestionario del alumnado especifica cuatro factores que explican el $63.436 \%$ de la varianza. Ver la Tabla 3.

Tabla 3. Matriz de componentes rotados del análisis factorial de las respuestas del profesorado del aula ordinaria al cuestionario de adaptación escolar. Educación primaria. Curso 2006-2007.

\begin{tabular}{|c|c|c|c|c|}
\hline & \multicolumn{4}{|c|}{ Componentes } \\
\hline & 1 & 2 & 3 & 4 \\
\hline Se muestra seguro & & ,696 & & \\
\hline Se muestra abierto & ,605 & & & \\
\hline Se muestra agresivo & & &,- 774 & \\
\hline Falta a clase & & & & \\
\hline Se muestra bien adaptado al aula & & 779 & & \\
\hline Se muestra bien adaptado al centro & & ,755 & & \\
\hline Tiene interés por aprender & ,812 & & & \\
\hline Mantiene la atención & ,802 & & & \\
\hline Participa en las tareas que se proponen & ,757 & & & \\
\hline Se esfuerza por hacer bien el trabajo & ,794 & & & \\
\hline Sabe pedir ayuda & ,591 & & & \\
\hline Tiene los hábitos de trabajo básicos & ,797 & & & \\
\hline $\begin{array}{l}\text { Tiene las estrategias suficientes para afrontar las } \\
\text { actividades del aula }\end{array}$ & ,744 & & & \\
\hline Se relaciona con facilidad con los compañeros & & 654 & & \\
\hline $\begin{array}{l}\text { Se relaciona, únicamente, con los compañeros de la } \\
\text { propia cultura }\end{array}$ & & & & ,873 \\
\hline $\begin{array}{l}\text { Se relaciona, únicamente, con los compañeros del } \\
\text { aula de acogida }\end{array}$ & & & & ,874 \\
\hline $\begin{array}{l}\text { Muestra actitudes de rechazo hacia determinados } \\
\text { compañeros }\end{array}$ & & &,- 696 & \\
\hline Es bien aceptado por los compañeros en el aula & & ,686 & & \\
\hline Es bien aceptado por los compañeros en el recreo & & ,709 & & \\
\hline Tiene buena relación con el profesorado & & ,503 & & \\
\hline Actúa con autonomía & ,663 & & & \\
\hline Respeta las opiniones del profesorado & & & 675 & \\
\hline $\begin{array}{l}\text { Tiene buena relación con otros profesionales del } \\
\text { centro }\end{array}$ & & & ,485 & \\
\hline
\end{tabular}

Este análisis factorial reproduce los componentes que hemos enunciado en el apartado anterior. Es decir el primer componente es "esfuerzo individual y hábitos de trabajo del alumnado". El segundo y el tercer componente son, respectivamente, "satisfacción del alumnado" y "malestar del alumnado" y, finalmente, el cuarto componente es "preferencias en la relación 
con los/las compañeros/as". El primer componente explica el $41.315 \%$ de la varianza, el segundo el $9.160 \%$, el tercero el $7.295 \%$ y el cuarto el $5.666 \%$.

Los componentes del análisis factorial de las respuestas del alumnado al cuestionario y la familia lingüística

El gráfico 1 muestra la relación entre el primer componente ("satisfacción en el aula ordinaria y esfuerzo individual") y la variable "familia lingüística".

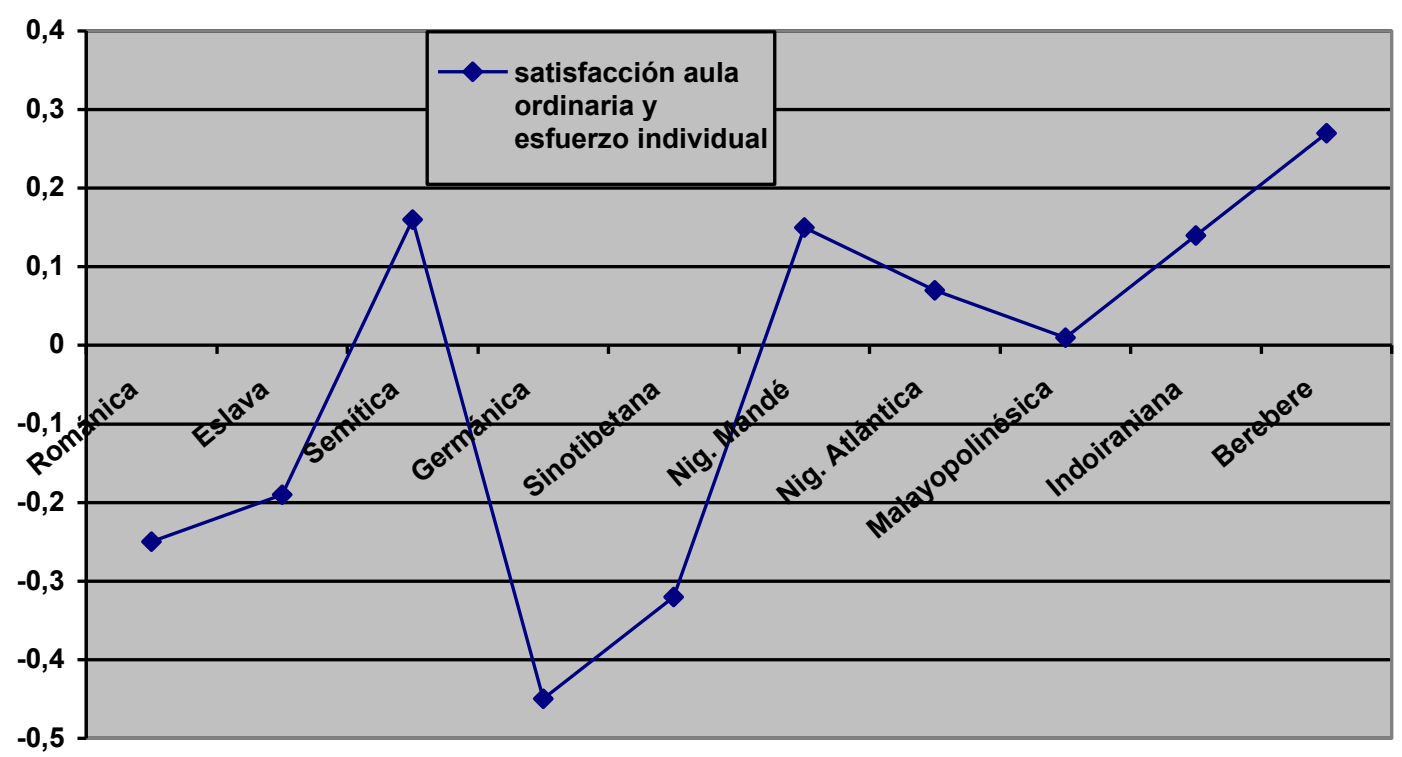

$\mathrm{F}=14,656 ; \mathrm{gl}=9 ; \mathrm{p}<0,000$

\section{Gráfico 1. Satisfacción en el aula ordinaria y esfuerzo individual del alumnado y familia lingüís- tica. Educación primaria. Curso 2006-2007.}

El alumnado que habla una lengua de la familia germánica o una lengua de la familia sinotibetana aparece como el más insatisfecho en el aula ordinaria y el que muestra una autopercepción de esforzarse poco en el trabajo escolar. Al contrario, el alumnado amazig es el que manifiesta más satisfacción y una mayor autopercepción sobre su esfuerzo en el trabajo escolar.

El alumnado que tiene como lengua inicial una lengua de la familia románica o eslava tiene una autopercepción negativa de su implicación en el trabajo escolar y muestra insatisfacción en el aula ordinaria a diferencia del alumnado que habla una lengua de la familia indoiraniana, semítica o nigerocongolesa que muestra grados razonables de satisfacción y auto- 
percepción positiva de su implicación en el trabajo escolar. Si embargo, si bien las diferencias no son significativas con el alumnado latinoamericano, el alumnado rumano muestra una autopercepción ligeramente positiva $(X=0.0269 ; \mathrm{dt}=0.968)$.

Finalmente, el alumnado que habla una lengua de la familia malayopolinésica está un poco por debajo de la línea base y, por tanto, sus actitudes se pueden considerar más cerca de una posición neutra.

La relación entre el segundo componente ("profesorado") y la variable "familia lingüística" es significativa $(\mathrm{F}(9,6240)=4.941 ; \mathrm{p}<0.001)$ y muestra que la percepción subjetiva del alumnado hacia el profesorado es negativa entre el alumnado con una lengua sinotibetana $(\mathrm{M}=-0.0844035 ; \mathrm{dt}=1.1023)$, románica $(\mathrm{M}=-0.0540075 ; \mathrm{dt}=1.0016)$ y malayopolinésica $(\mathrm{M}=-$ 0.0986318; $\mathrm{dt}=1.2284)$. El alumnado que habla una lengua eslava tiene una actitud ligeramente negativa $(\mathrm{M}=-0.020868 ; \mathrm{dt}=1.1441)$ y el resto tiene una actitud positiva, de la cual hay que destacar la actitud del alumnado con una lengua indoiraniana $(\mathrm{M}=0.1598933$; $\mathrm{dt}=0.9208)$ y nigerocongolesa atlántica $(\mathrm{M}=0.1577740 ; \mathrm{dt}=0.8799)$.

Hay diferencias significativas entre la percepción del alumnado de lengua castellana y de lengua rumana $(\mathrm{F}(1,3373)=24.004 ; \mathrm{p}<0.001)$ en el sentido de una clara percepción positiva por parte del alumnado rumano $(\mathrm{M}=0.1368$; $\mathrm{dt}=1.0078)$ y una percepción negativa por parte del alumnado hispano $(\mathrm{M}=-0.0868 ; \mathrm{dt}=0.9876)$

El gráfico 2 muestra la relación entre el tercer componente (“aprendizaje”) y la variable "familia lingüística". 


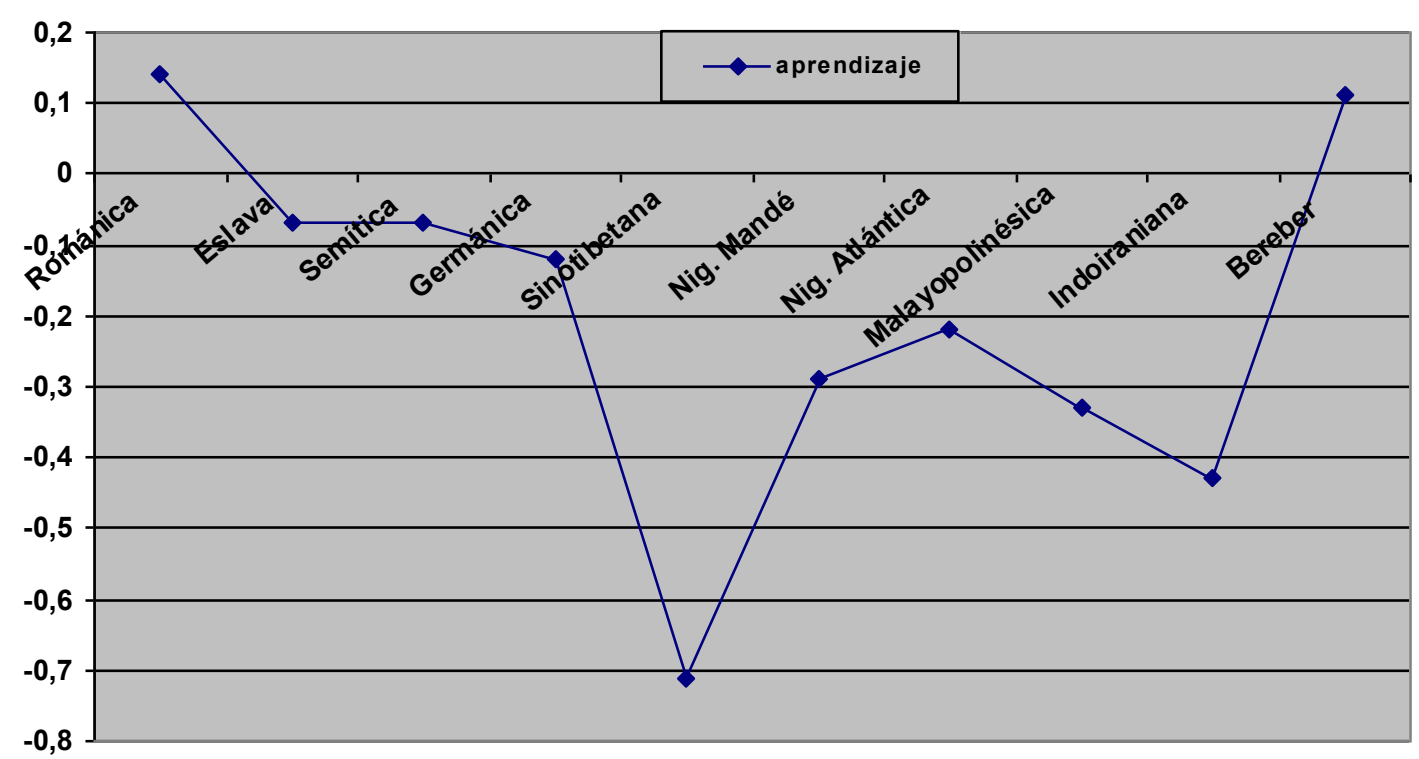

$\mathrm{F}=38,654 ; \mathrm{gl}=9 ; \mathrm{p}<0,001$

Gráfico 2. Aprendizaje y familia lingüística. Educación primaria. Curso 2006-2007.

Los resultados muestran que la mayoría del alumnado considera que no aprende o que aprende poco $(\mathrm{M}=-0.0000394 ; \mathrm{dt}=1.000597)$. Únicamente el alumnado que habla una lengua románica o amazig considera que está aprendiendo.

Hay diferencias significativas entre el alumnado de lengua castellana y el alumnado de lengua rumana $(\mathrm{F}(1,3373)=6,680 ; \mathrm{p}<0,01)$ en el sentido de una mejor autopercepción del proceso de aprendizaje por parte del alumnado rumano $(\mathrm{M}=0.2276$; $\mathrm{dt}=0.9154)$ que del alumnado latinoamericano $(\mathrm{M}=0.1152 ; \mathrm{dt}=0.9496)$.

La percepción del alumnado que habla una lengua sinotibetana sobre su proceso de aprendizaje muestra que la mayoría de este alumnado responde "nada" a las preguntas del cuestionario que forman parte de este componente.

A la percepción subjetiva que el alumnado con una lengua sinotibetana tiene sobre su proceso de aprendizaje se puede añadir la del alumnado que habla una lengua indoiraniana. La percepción del alumnado con una lengua sinotibetana se corresponde con las actitudes mostradas en relación al primer componente, pero no es así en el caso del alumnado con una lengua indoiraniana, el cual tenía actitudes abiertamente positivas respecto a su proceso de implicación en el aula ordinaria. 
La percepción del alumnado que habla una lengua semítica, malayopolinésica y nigerocongolesa mandé mostraba actitudes positivas sobre su implicación en el trabajo escolar del aula ordinaria. Sin embargo, en este componente, su percepción subjetiva es negativa.

La relación entre el cuarto componente ("satisfacción en el aula de acogida") y la variable "familia lingüística" también es significativa $(F(9,6240)=9.860 ; p<0.001)$. Los grados máximos de satisfacción en el aula de acogida los expresa el alumnado con una lengua semítica, nigerocongolesa mandé, malayopolinésica, indoiraniana y amazig, mientras que el resto del alumnado se muestra insatisfecho, especialmente el alumnado con una lengua nigerocongolesa atlántica.

Entre el alumnado rumano y el alumnado latinoamericano hay diferencias significativas $(\mathrm{F}(1,3373)=4.734 ; \mathrm{p}<0.030)$. El alumnado rumano está más satisfecho en el aula de acogida $(\mathrm{M}=0.0258 ; \mathrm{dt}=0.8697)$ que el alumnado latinoamericano, el cual está insatisfecho $(\mathrm{M}=-$ $0.0729 ; \mathrm{dt}=1.0076)$.

El gráfico 3 muestra la relación entre los tres últimos componentes, los que se refieren a la relación con los iguales, y la variable "familia lingüística”.

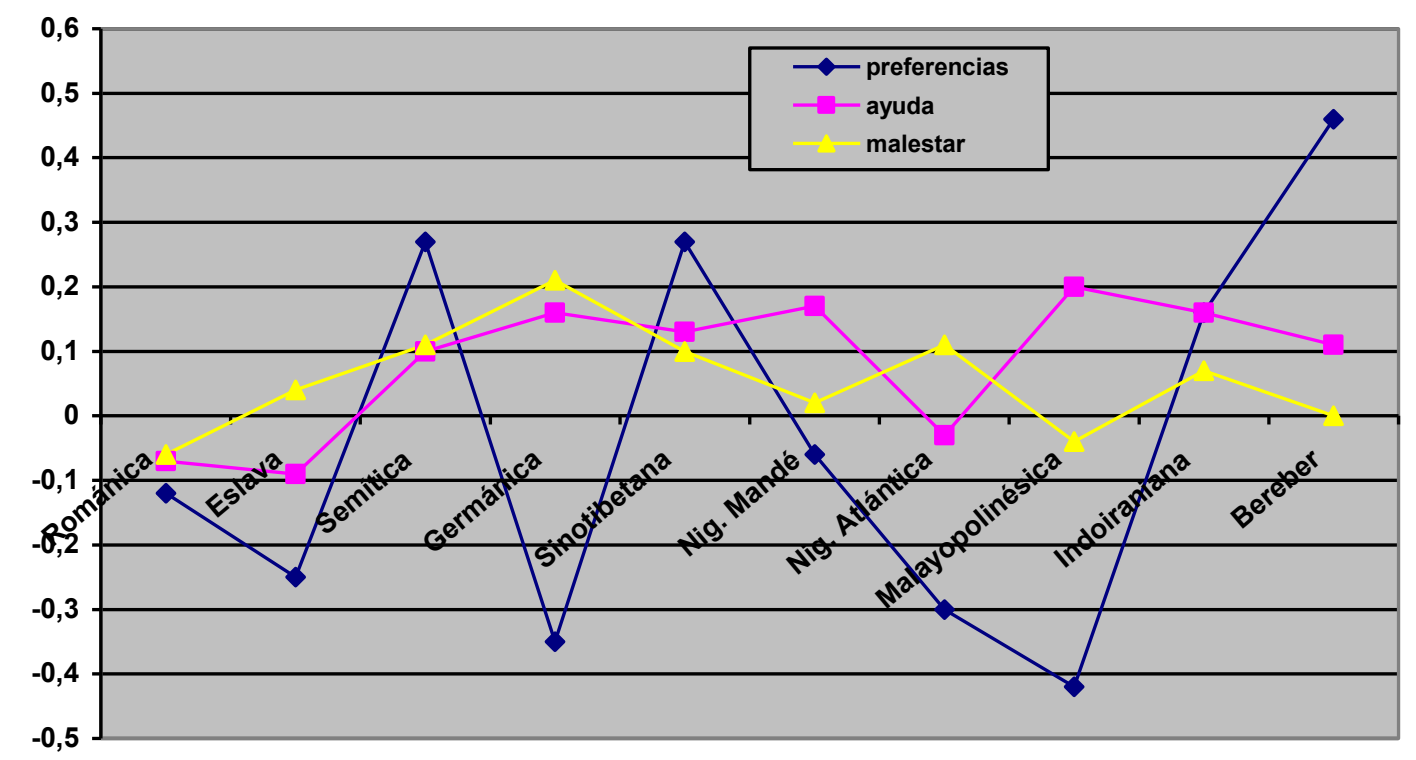

$(F(9,5644)=31.291 ; p<0.001$ para el quinto componente; $F(9.5644)=6$ '359; $p<0.001$ para el sexto componente; $\mathrm{F}(9.5644)=4,210 ; \mathrm{p}<0.001$ para el séptimo componente)

Gráfico 3. Preferencias, ayuda y malestar con los/las compañeros/as y familia lingüística. Educación primaria. Curso 2006-2007. 
Respecto a los tres componentes analizados globalmente, el alumnado de las diferentes familias lingüísticas se comporta homogéneamente, excepto el de lengua amazig, malayopolinésica, nigerocongolesa atlántica y germánica. Las discrepancias aparecen entre el primer componente ("preferencias en la relación con los/las compañeros/as") y los otros dos componentes ("ayuda y compañeros" y "malestar con los/las compañeros/as"). La única explicación coherente se puede ofrecer respecto al alumnado amazig. Así, tiene un valor alto en el quinto componente ("preferencias en las relaciones con los/las compañeros/as") lo que significa que busca relaciones con compañeros de su cultura o del aula de acogida $y$, por tanto, puede tener una percepción de que hay otros compañeros que le molestan y, por eso, su valor es cero, pero en los otros casos esta explicación no sirve ya que tienen valores muy negativos en el quinto componente ("preferencias en la relación con los/las compañeros/as") lo que quiere decir que se relacionan con todos los compañeros y, a la vez, positivos en el séptimo componente ("malestar con los/las compañeros/as") lo que significa que llevan mal las relaciones con una parte de sus iguales.

El alumnado con una lengua románica es el más homogéneo, probablemente porque es mayoría en las aulas. En este sentido, tiende a relacionarse con todo el mundo, no percibe una mala relación con el resto de compañeros y no percibe ayudas especiales de sus iguales. El alumnado que habla una lengua eslava tiene percepciones parecidas, si bien un poco más acentuadas respecto a una buena relación con todos los compañeros.

Hay diferencias significativas entre el alumnado rumano y el alumnado latinoamericano en el componente "malestar con los/las compañeros/as" (F $(1,3373)=14.286 ; p<0.001)$. El alumnado rumano tiene un valor positivo $(\mathrm{M}=0.0697$; $\mathrm{dt}=1.0186)$ y el alumnado latinoamericano negativo $(\mathrm{M}=-0.1012 ; \mathrm{dt}=0.9745)$. No obstante, respecto al quinto componente, el alumnado rumano no muestra preferencias especiales con grupos de compañeros $(M=-0.0559734$; $\mathrm{dt}=0.9204)$, si bien su media es inferior a la del alumnado latinoamericano $(\mathrm{M}=-0.1090$; $\mathrm{dt}=0.8443)$.

El alumnado que habla una lengua sinotibetana, nigerocongolesa mandé, semítica e indoiraniana tiene percepciones parecidas. De una parte, tiene tendencia a relacionarse con compañeros de la propia cultura o del aula de acogida y, en este sentido, percibe que tiene una relación ligeramente mala con una parte del resto de iguales. Pero, de la otra, siente que es apoyado tanto en el aula de acogida como en el aula ordinaria por compañeros de clase. 
Los componentes del análisis factorial de las respuestas del profesorado del aula de acogida al cuestionario y la familia lingüística

El gráfico 4 muestra la relación entre el primer componente ("esfuerzo individual y hábitos de trabajo del alumnado") y la variable "familia lingüística".

El gráfico que describe la percepción que muestra el profesorado del aula de acogida sobre la implicación del alumnado tiene poco que ver con la percepción que el alumnado tiene sobre esta cuestión. Así, las percepciones tanto positivas como negativas del alumnado se transforman, por parte del profesorado del aula de acogida, en lo contrario. Únicamente hay una cierta coincidencia respecto al alumnado que habla una lengua nigerocongolesa atlántica y malayopolinésica. En el resto, el resultado es distinto. Así, hay diferencias entre las percepciones que muestra el alumnado de lengua eslava, sinotibetana, semítica, nigerocongolesa mandé y indoiraniana y las percepciones sobre este alumnado del profesorado del aula de acogida.

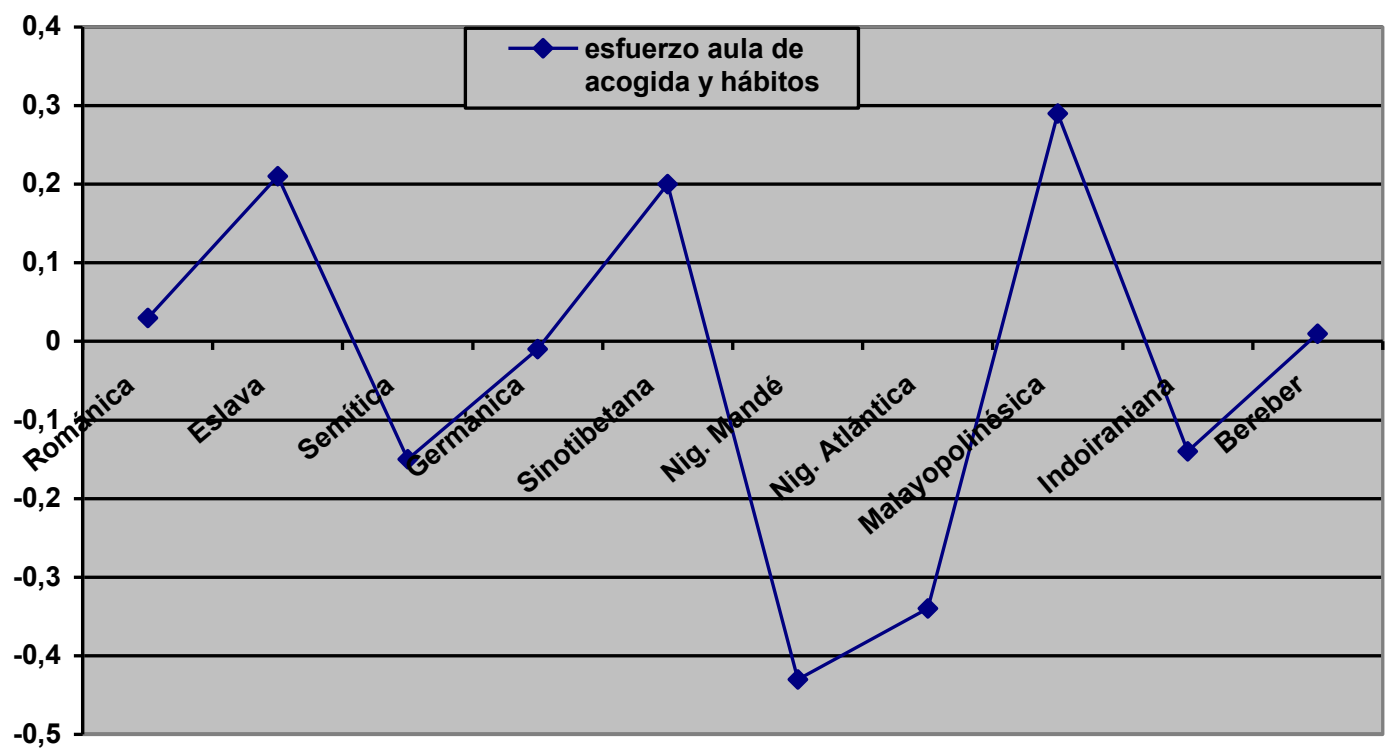

$\mathrm{F}=10,019 ; \mathrm{gl}=9 ; \mathrm{p}<0,000$

Gráfico 4. Esfuerzo individual y hábitos de trabajo del alumnado y familia lingüística. Profesorado aula de acogida. Educación primaria. Curso 2006-2007.

Los perfiles del segundo ("satisfacción del alumnado") y del tercer componente ("malestar del alumnado") y la familia lingüística son significativos respectivamente $\{\mathrm{F}$ $(9,6837)=19.006 ; p<0.001$ y $F(9,6837)=15.881 ; p<0.001\}$ y muestran que, excepto en el caso del alumnado que habla una lengua indoiraniana y, en parte, del alumnado que habla una len- 
gua malayopolinésica, los resultados sobre la satisfacción y el malestar del alumnado percibido por el profesorado del aula de acogida son muy coherentes y coinciden con las percepciones que tiene el alumnado sobre esta cuestión.

El gráfico 5 muestra el perfil del cuarto componente ("relación con los/las compañeros/as") y la variable continente.

Independientemente de la percepción del alumnado, la percepción subjetiva del profesorado del aula de acogida sobre las relaciones entre el alumnado muestra que la mayoría de los africanos y de los asiáticos son percibidos con problemas (poca aceptación del resto de compañeros, rechazo, etc.) respecto a sus relaciones, mientras que todo el alumnado europeo y latinoamericano es percibido sin problemas de integración.

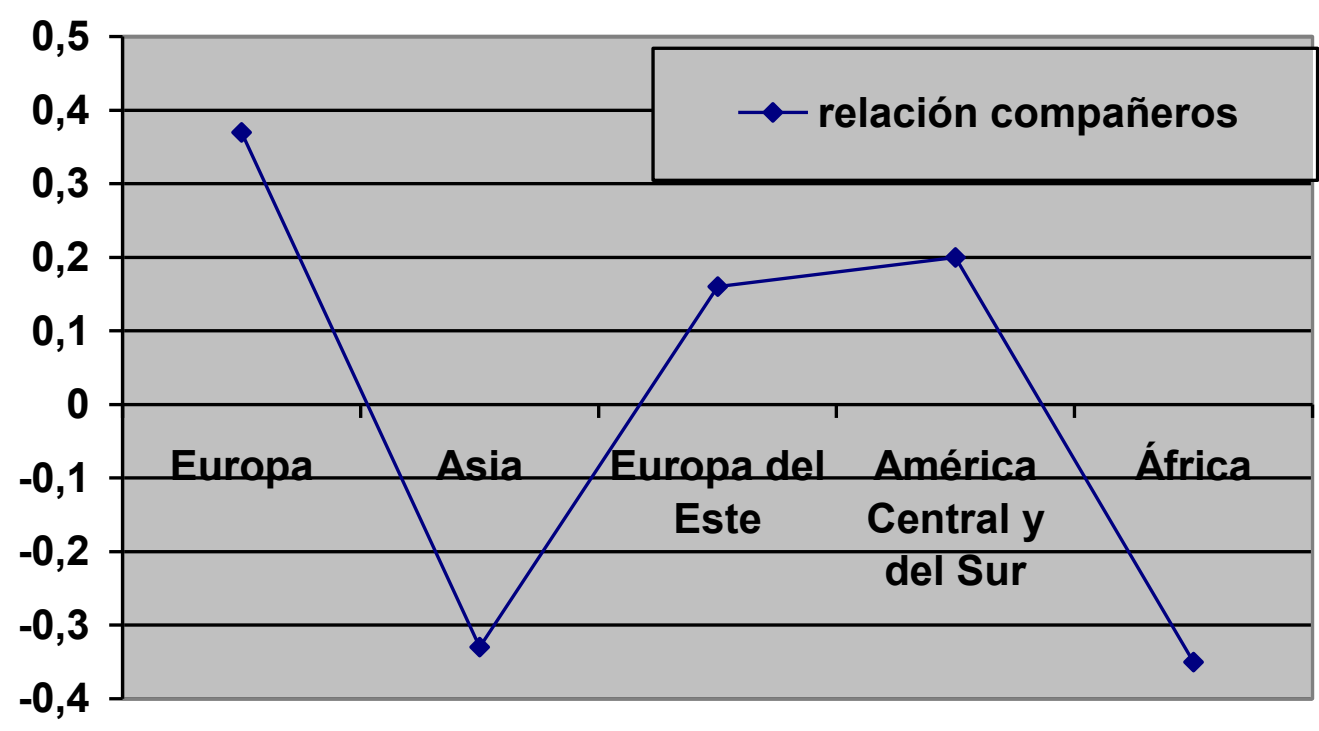

$F(6,5717)=90.286 ; p<0.001)$

Gráfico 5. Relación con los compañeros y continente. Profesorado aula de acogida. Educación primaria. Curso 2006-2007.

Hay diferencias significativas en el test de Scheffe entre los europeos, que son los mejor percibidos, y el resto de grupos $(\mathrm{p}<0.001$ con Asia y África; $\mathrm{p}<0.016$ con Europa del Este y $\mathrm{p}<0.043$ con América Central y del Sur) y entre los africanos y los asiáticos con el resto de grupos $(\mathrm{p}<0.001)$. 
Los componentes del análisis factorial de las respuestas del profesorado del aula ordinaria al cuestionario y la familia lingüistica

El gráfico 6 muestra la relación entre el primer componente ("esfuerzo individual y hábitos de trabajo del alumnado") y la variable "familia lingüística".

De manera más atenuada que el profesorado del aula de acogida, el profesorado del aula ordinaria tampoco coincide con las percepciones que tiene el alumnado de su implicación en el aula ordinaria. Así, de nuevo aparecen invertidas las percepciones, excepto en el caso del alumnado que habla una lengua románica o malayopolinésica.

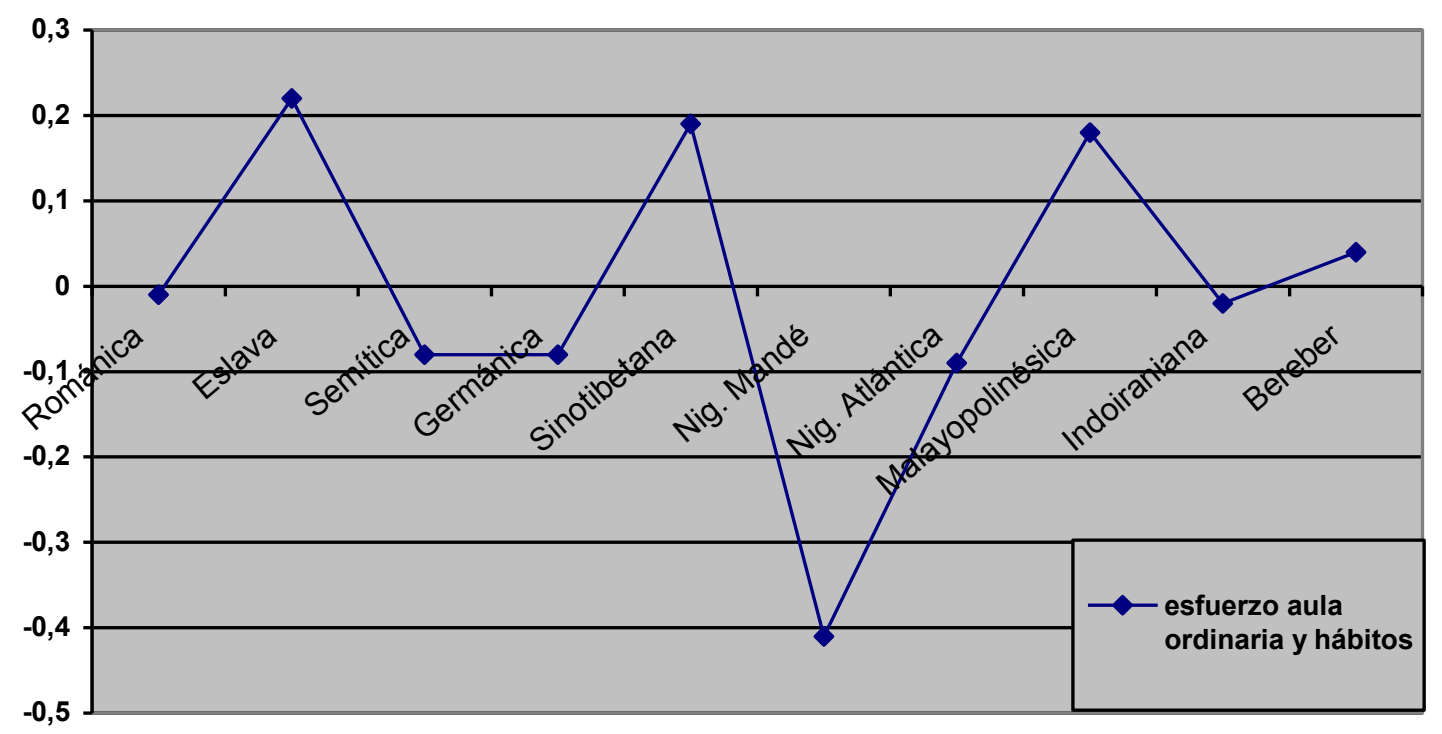

$\mathrm{F}(9,5644)=5.475 ; \mathrm{p}<0.001$

Gráfico 6. Esfuerzo individual y hábitos de trabajo del alumnado y familia lingüística. Profesorado aula ordinaria. Educación primaria. Curso 2006-2007.

El perfil de los componentes segundo ("satisfacción del alumnado") y tercero ("malestar del alumnado") y la familia lingüística son significativos respectivamente $\{\mathrm{F}$ $(9,6606)=7.643 ; \mathrm{p}<0.001$ y $\mathrm{F}(9,6606)=17.801 ; \mathrm{p}<0.001\}$. Ambos coinciden, si exceptuamos al alumnado con una lengua malayopolinésica y, en parte, al alumnado que habla una lengua semítica, con los perfiles de los mismos componentes del cuestionario del profesorado del aula de acogida. Son muy coherentes y, en general, reflejan las percepciones del alumnado. Las percepciones del profesorado del aula ordinaria respecto al alumnado con una lengua semítica se pueden explicar por la percepción negativa sobre su satisfacción $(\mathrm{M}=-0.1262313$; 
$\mathrm{dt}=0.984623$ ). Así, dado que, en el tercer componente, en los ítems relacionados con las y los profesionales del centro (profesorado y otros), la puntuación es baja y que, además, el alumnado de lengua semítica tiene una puntuación elevada en los ítems que operan negativamente (por ejemplo, se muestra agresivo) hace que, en conjunto, la puntuación sea baja $(\mathrm{M}=-$ $0.2721901 ; \mathrm{dt}=1.108067)$.

Finalmente, el gráfico 7 muestra las relaciones entre el cuarto componente ("preferencias en la relación con los/las compañeros/as") y la variable continente.

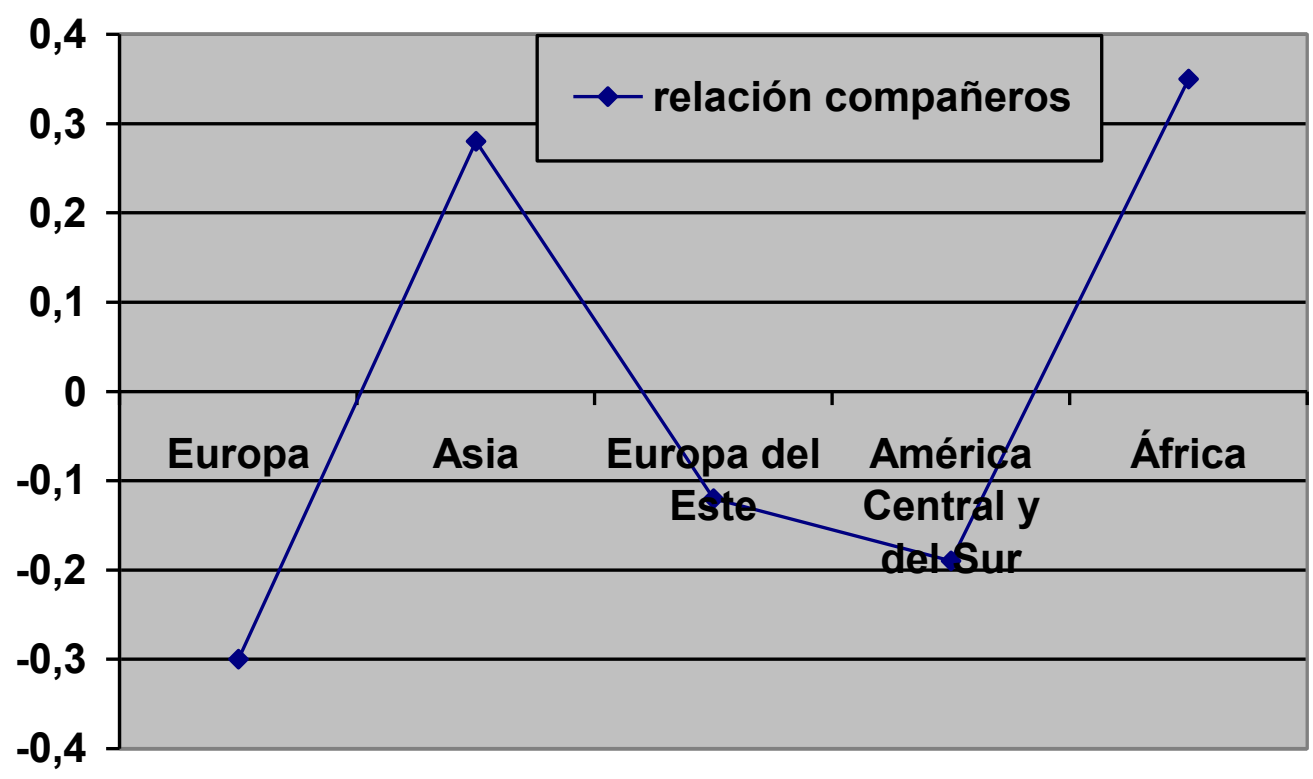

$\mathrm{F}(6,5717)=71.991 ; \mathrm{p}<0.001$

Gráfico 7. Preferencias en la relación con los compañeros y familia lingüística. Profesorado aula ordinaria. Educación primaria. Curso 2006-2007.

El análisis de varianza mediante la variable "continente" reproduce prácticamente la misma percepción que la que tiene el profesorado del aula de acogida, de manera que africanos y asiáticos están en un lado y el resto en el otro. En este caso, los valores positivos se refieren a las preguntas de este componente que son distintas a las del cuarto componente de las respuestas del profesorado del aula de acogida. En este componente, las preguntas que se incluyen son "prefiere relacionarse sólo con el alumnado de su propia cultura" o "del aula de acogida", mientras que en el cuarto componente de las respuestas del profesorado del aula de 
acogida las preguntas que se incluyen son del tipo "es bien aceptado por sus iguales en el aula" o "en el recreo". Por eso, ambos gráficos están invertidos pero expresan el mismo tipo de actitudes por parte del profesorado.

Únicamente hay diferencias significativas entre los asiáticos y los africanos con el resto de grupos $(\mathrm{p}<0.001)$.

\section{Discusión y conclusiones}

Las respuestas del alumnado extranjero de las aulas de acogida de primaria de Cataluña y de su profesorado al cuestionario de adaptación escolar muestran importantes discrepancias sobre la percepción que unos y otros tienen sobre la implicación de dicho alumnado y de sus relaciones.

Las actitudes del alumnado extranjero sobre su implicación y satisfacción con el contexto escolar son muy diferentes de acuerdo con las agrupaciones realizadas mediante su lengua propia. En un extremo tenemos al alumnado africano de lengua árabe o amazig y, en el otro, al alumnado que habla una lengua sinotibetana o germánica. Entre dichos extremos, encontramos al resto del alumnado, del cual cabe destacar al alumnado latinoamericano que representa algo más del 40\% del alumnado extranjero y que mantiene una actitud ligeramente negativa.

La inmensa mayoría del alumnado extranjero de las aulas de acogida de la educación primaria de Cataluña ha tenido previamente una escolarización regular (Siqués, 2008) y, por tanto, podemos suponer que sus grados de satisfacción o insatisfacción con el actual contexto escolar se construyen, entre otras cosas, a partir de sus percepciones anteriores del contexto escolar en su país de origen y de sus creencias sobre el proceso de aprendizaje. Por eso, no es extraño que el alumnado magrebí muestre actitudes positivas, especialmente el alumnado amazig, mientras que el alumnado que habla una lengua sinotibetana muestre una profunda insatisfacción. El sistema educativo del Magreb no es muy distinto en su organización del sistema educativo de Cataluña, pero la práctica educativa es bastante más rígida y, además, sus recursos humanos y materiales son muy inferiores a los del sistema educativo catalán. Además, en el caso del alumnado de lengua amazig, su lengua es abiertamente rechazada y se impone el dariya como única lengua escolar (Colectivo IOÉ, 1996). Esta explicación apoya 
que las actitudes del alumnado magrebí hacia el profesorado sean muy positivas. Por lo que se refiere al sistema educativo de China, la manera de ejercer la docencia $y$, en consecuencia, el aprendizaje del alumnado, dista enormemente de nuestros sistemas educativos. En este caso, el aprendizaje se apoya exclusivamente en la transmisión de informaciones por parte del profesorado y se basa en la repetición y la memorización, lo cual se aleja enormemente de la tradición pedagógica de Cataluña que, en muchos casos, se apoya en el trabajo cooperativo y en diversas variantes del aprendizaje por descubrimiento. (Pérez-Millans, 2006)

El alumnado de lengua germánica también manifiesta importantes grados de insatisfacción y de falta de implicación en el contexto escolar. Una posible explicación de estas actitudes puede estar en el contraste entre su estatus escolar previo y el actual. En general, las familias de este alumnado son de nivel socioprofesional y educativo alto. Sin embargo, su "nueva" escolarización se realiza en contextos escolares en los que una buena parte del alumnado tiene un origen extranjero que, en los países de habla germánica, está escolarmente segregado. En el componente que hemos denominado "malestar con el resto del alumnado", el alumnado de lengua germánica es el que tiene valores más altos, lo cual puede indicar el rechazo a una parte del alumnado, de modo que su insatisfacción escolar reproduce fundamentalmente su insatisfacción con una parte de sus compañeros y compañeras. Otro dato que apoya esta explicación es la existencia de actitudes positivas de este alumnado hacia el profesorado, mientras que las del alumnado de lengua sinotibetana son abiertamente negativas.

Sin embargo, la percepción del profesorado sobre la implicación y la satisfacción del alumnado extranjero en el contexto escolar son, en algunos casos, abiertamente contradictorias. El alumnado de lengua sinotibetana y su profesorado coinciden en percibir mutuamente un importante grado de insatisfacción. Pero, a diferencia de lo que afirma el alumnado chino, su profesorado lo tiene en alta estima y considera que está muy implicado en las actividades escolares y, en consecuencia, está aprendiendo. Es justamente la percepción inversa sobre el alumnado magrebí. De una parte, el profesorado valora la satisfacción de este alumnado más negativamente que la autopercepción que éste manifiesta y, de la otra, considera, a diferencia claramente de las actitudes que manifiesta el alumnado, que está poco implicado en el contexto escolar. Y, en relación al alumnado de lengua germánica, el profesorado reconoce en parte su insatisfacción, pero mantiene actitudes positivas hacia su implicación en el trabajo escolar. 
Probablemente, las relaciones más estrechas entre la autopercepción del alumnado y la percepción del profesorado se producen con el alumnado de lengua rumana y el de lengua eslava, mientras que con el alumnado de lengua castellana aparecen algunas discrepancias. En relación al alumnado rumano, tanto éste como su profesorado tienen valoraciones mutuas positivas y, además, el alumnado rumano está satisfecho y se considera implicado. Ambas cosas son reconocidas por su profesorado. En cambio, el alumnado hispano muestra una leve insatisfacción, especialmente en el aula de acogida, y una actitud neutra hacia el aprendizaje escolar, si bien su autoestima es alta y considera que está aprendiendo. El profesorado coincide en esta segunda apreciación, si bien considera que está algo más implicado y satisfecho que lo que afirma este alumnado. Probablemente, las manifestaciones subjetivas del alumnado latinoamericano se relacionan con el "descubrimiento" del catalán como lengua de enseñanza y aprendizaje. Así, a los aspectos negativos ligados al proceso migratorio, se añade el aprendizaje, no esperado, de una nueva lengua.

Por último, las percepciones del alumnado africano de lengua nigerocongolesa y de su profesorado son semejantes a las percepciones mutuas que tienen el alumnado de lengua semítica y su profesorado. La diferencia estriba en que el profesorado percibe más negativamente la implicación escolar del alumnado africano de lengua nigerocongolesa que el de lengua semítica.

Estos datos muestran los retos que tiene que afrontar la educación intercultural. De hecho, no podemos afirmar que las percepciones del profesorado sobre el alumnado extranjero reproducen las percepciones sociales sobre las personas extranjeras, pero en buena parte coinciden. Así, existe una estrecha relación entre lo que manifiesta el profesorado y aquello que se piensa socialmente sobre las "facilidades" de integración de las personas extranjeras. Africanos y asiáticos, con algún matiz que introduce el profesorado del aula ordinaria, son percibidos por el profesorado con más dificultades de integración que el resto del alumnado extranjero. Igualmente, la distinta percepción del profesorado sobre la implicación escolar del alumnado magrebí y chino coincide claramente con los prejuicios sociales que existen sobre esta cuestión (Defensor del Pueblo, 2003).

Los datos que hemos expuesto revelan las dificultades de la educación intercultural y quizás también las dificultades de una parte del alumnado de origen extranjero en el contexto escolar. Es evidente que los resultados escolares no se pueden explicar únicamente a partir de las 
percepciones y expectativas del profesorado sobre el alumnado, sino que intervienen numerosos factores. Además, las creencias del profesorado se construyen a partir de índices muy distintos, algunos de ellos claramente relacionados con el entorno social (por ejemplo, a través de la influencia educativa de los medios de comunicación). Por eso, las explicaciones que establecen relaciones causales entre las percepciones que tiene el profesorado del alumnado y sus resultados escolares son, a nuestro entender, difíciles de mantener, aunque creemos que dichas percepciones pueden tener una influencia que ciertamente no abordamos en este trabajo.

\section{Referencias}

Banks, J.A. y Banks, C.A.M. (eds.) (1989). Multicultural Education: Issues ans Perspectives. New York: John Wiley.

Besalú, X. (2006). El plan para la lengua y la cohesión social (Plan LIC) del gobierno de Cataluña. Revista Interuniversitaria de Formación del Profesorado, 20(2), 45-68.

Besalú, X. y Vila, I. (2005). Consolidar la cohesió social, l'educació intercultural i la llengua catalana. Caixa d'eines, 01, 6-33.

Besalú, X. y Vila, I. (2007). La buena educación. Libertad e igualdad en la escuela del Siglo XXI. Madrid: Los Libros de la Catarata/MEC.

Coenders, M., Scheepers, P., Sniderman, P. y Verbeck, G. (2001). Blatant and subtle prejudice: dimensions, determinants, and consequences? European Journal of Social Psycho$\log y, 31(3), 281-297$.

Colectivo IOÉ (1996). La educación intercultural a prueba: Hijos de marroquíes en la escuela. Granada: Laboratorio de Estudios Interculturales.

Cummins, J. (2000). Language, Power and Pedagogy. Clevedon: Multilingual Matters. (Traducción castellana. Lenguaje, poder y pedagogía. Madrid: Morata, 2002)

Defensor del Pueblo (2003). La escolarización del alumnado inmigrante en España: análisis descriptivo y estudio empírico. Madrid.

Departament d'Educació (2004). Plan para la Lengua y la Cohesión Social. Educación e Inmigración. www.xtec.cat/lic/. Consulta el 15 de mayo de 2008.

European Monitoring Centre on Racism and Xenophobia (2005). Majorities' Attitudes Towars Minorities: Key Findings from the Eurobarometer and the European Social Survey. Viena: Manz Crossmedia GmbH\&CO KG. 
Fernández-Castillo, A. (2005). Multiculturalidad en Contextos Educativos y de Desarrollo: Relevancia de Variables Psicosociales. Electronic Journal of Research in Educational Psychology, 3(1), 181-204.

IDESCAT (2008). La natalitat a Catalunya l'any 2006. Nota de prensa del 21 de febrero.

INE (2008). Encuesta nacional de inmigrantes. Avance de resultados. Notas de prensa, 22 de mayo.

León del Barco, B., Mira, A.R. y Gómez-Carroza, T. (2007). Evaluación de las opiniones sobre la inmigración y la interculturalidad en la escuela de alumnos de Magisterio. Electronic Journal of Reserach in Educational Psychology, 5(2), 259-282.

Mcintosh, P. (1990). White privilege: unpacking the invisible knapsack. Independent School, 31-36.

Mcintyre, A. (1997). Constructing an image of a white teacher. Teachers College Record, 98 (4), 1-15.

Pérez-Millans, M. (2006). Spanish Education and Chinese Immigrants in a New Multicultural Context: Cross-cultural and Interactive Perspectivas in the Study of Language Teaching Methods. Journal of Multicultural Discourses, 1(1), 60-85.

Primo, L.E., Quaglia, R. y Schavo, E. (2008). Prejuicio en la escuela: una investigación con profesores de educación primaria. European Journal of Education and Psychology, 1(3), 27-37.

Serra, J.M. (2006). El plan de lengua y cohesión social en Cataluña: primeros datos de una investigación. Cultura y Educación, 18(2), 159-172.

Siqués, C. (2008). Les aules d'acollida d'educació primària de Catalunya : descripció i avaluació dels resultats. Tesis Doctoral. Departamento de Psicología. Universidad de Girona.

Sleeter, C.E. (1992). Resisting racial awareness: how teachers understand the social order from their racial, gender and social locations, Educational Foundations, 7, 7-31.

Solomona, P.R., Portelli, J.P., Beverly-Jean, D., Campbell, A. (2005). The discourse of denial: how white teacher candidates construct race, racism amn "white privilege". Race Ethnicity and Education, 8(2), 147-169.

Verbeck, G., Scheepers, P. y Felling, A. (2002). Attitudes and behavioural intentions towards ethnic minorities: an empirical test of several theoretical explanations for the Dutch case. Journal of Ethnic and Migratioin Studies, 28(2), 197-219.

Vila, I. Perera, S. y Serra, J.M. (2005). L'avaluació de les aules d'acollida. Curs 2004-2005. Documento no publicado. Servei d'Ensenyament del Català. Generalitat de Catalunya. 
Vila, I., Perera, S. y Serra, J.M. (2006). Les aules d'acollida en l'educació primària. Algunes dades del curs 2004-2005. Caixa d'Eines, 04, 40-55.

Vila, I., Canal, I., Mayans, P., Perera, S., Serra, J.M. y Siqués, C. (2007). Les aules d'acollida de l'educació primària i secundària obligatòria de Catalunya: un estudi comparatiu. Catalan Review, 21, 351-380.

Vila, I., Canal, I. , Mayans, P., Perera, S. , Serra, J.M., Siqués, C. (en prensa). Las aulas de acogida de la educación primaria de Cataluña el curso 2005-2006: sus efectos sobre el conocimiento de catalán y la adaptación escolar, Infancia y Aprendizaje 32 (3).

Weiner, L. (2000). Research in the 90s: Implications for urban teacher preparation. Review of Educational Research, 70(3), 369-406. 